Original Research Article

\title{
Perfusion index and Plethysmographic variability index values in spontaneously breathing clinically stable term neonates in room air in the first 10 days of life
}

\author{
Ashok Raja $\mathbf{J}^{1 *}$, Balasankar $\mathbf{S}^{2}$, Mathiarasan $\mathrm{K}^{3}$ \\ ${ }^{1}$ DR J. Ashok Raja D.M. (Neonatology), DNB (Neo), Assistant Professor of Neonatology, ${ }^{2}$ Dr S. Balasankar MD, \\ DCH, Professor of Pediatrics, ${ }^{3}$ Dr. K. Mathiarasan MD, DCH, Director \& Professor of Pediatrics, Division of \\ Neonatology, Institute of Child Health and Research Centre, Madurai Medical College, Madurai, India.
}

Address for Correspondence: DR J. Ashok Raja, D.M. (Neonatology), DNB (Neo), Assistant Professor of Neonatology, Division of Neonatology, Institute of Child Health and Research Centre, Madurai Medical College. Email: ashokium@gmail.com.

\begin{abstract}
Background: Perfusion index (PI) and Pleth Variability Index (PVI) are newer pulseoximeter derived numerical non-invasive measures of Peripheral perfusion. PI gives real time changes in peripheral blood flow and PVI the dynamic changes in the PI that occur during the respiratory cycle. Reference values for neonatal population are very few and not yet published for Indian neonates. We undertook this study to establish the reference values for the first 10 days of life in term neonates. Methods: Prospective analytical study conducted at Division of Neonatology, Government Rajaji Hospital, Madurai Medical College. Inborn term neonates, spontaneously breathing in room air and clinically stable, without respiratory distress, on direct breast feeds or oral feeds are eligible for the study. PI, PVI values are recorded on the foot of neonates using Masimo Pulse oximeter for 15 minutes and data transferred to a computer and analyzed. Results: The Primary outcome median value of perfusion index is $1.4 \mathrm{IQR}(1.0,2.0)$ and Pleth variability index is $19.0 \mathrm{IQR}(15.0,25.0)$. Conclusions: The reference ranges for perfusion index and pleth variability index in healthy term Indian neonates are presented.
\end{abstract}

Keywords: Neonate, Newborn, Perfusion index (PI), Pleth Variability Index, Reference, Term

\section{Introduction}

Pulseoximeter is a non-invasive bedside equipment for assessing saturation and heart rate. Pulseoximetry uses the principle of preferential absorption of red light (R) by deoxyhemoglobin and infrared light (IR) by oxyhemoglobin. Pulseoximetry uses a light emitter with red and infrared LEDs and a photo detector at the opposite end, to receive the light passing through the measuring site. Newer generation Pulseoximeters display two other indices, Perfusion index (PI) to assess peripheral tissue perfusion, and pleth variability index (PVI) to assess the variability of perfusion index [1]. Perfusion index (PI) is a non invasive measure of real time changes in peripheral blood flow [1 - 4]. For the calculation of Perfusion

Manuscript received: $27^{\text {th }}$ July 2017

Reviewed: $6^{\text {th }}$ August 2017

Author Corrected: $14^{\text {th }}$ August 2017

Accepted for Publication: $21^{\text {st }}$ August 2017 index, the Infrared (IR) pulsatile signal is indexed against the non-pulsatile IR signal and expressed as a percentage. Pulsatile signal is due to a variable amount of light (AC) absorbed by the pulsating arterial inflow and non pulsatile signal (DC) is due to constant amount of light absorbed by the skin, other tissues and nonpulsatile blood.

The Infrared (IR) signal is used because it is less affected by changes in arterial saturation than the $\mathrm{R}$ signal $[1,2]$.

PI =IR pulsatile signal/IR nonpulsatile signal x100. During hypoperfusion, pulsatile signal due to arterial flow decreases whereas the nonpulsatile signal due to venous blood and other tissues does not change. Hence the ratio decreases and perfusion index is low. 
PI gives a dynamic numerical value for assessing peripheral perfusion [3]. It provides an objective measure and avoids potential observer bias in other methods of assessing peripheral perfusion like capillary refill time.

The other index, Pleth Variability Index (PVI) is a measure of dynamic changes in the PI. It is calculated by measuring changes in PI over a time interval where one or more complete respiratory cycles have occurred.

PVI $=($ PI Max - PI Min $) /$ PI Max x 100 [2, 5].

PVI therefore is displayed as a percentage. The lower the number, lesser the variability in the PI over a respiratory cycle. Increased PVI may occur in hypovolemia, shock, pericardial tamponade, pneumothorax and ventilator support, where the relationship between intravascular volume/ intrathoracic airway pressure increases [2].

Hence PI and PVI are potential non-invasive monitoring indices in many conditions in neonates like sepsis, shock, and to asses the severity of illness $[6,7]$. Establishing reference values in neonatal period is essential and it will open a new horizon for future studies. Studies regarding Reference values for neonatal population are very few [8-11] and not yet published for Indian neonates. Hence we undertook this study to establish the reference values for the first 10 days of life in term neonates, during which time most critically ill neonates are admitted.

\section{Methods}

Study design: Prospective analytical study

Setting: Tertiary care medical college hospital at South India, Division of Neonatology, Government Rajaji Hospital, Madurai Medical College from November 2013 to March 2014.

Inclusion criteria: Inborn term neonates, spontaneously breathing in room air and clinically stable, without respiratory distress, on direct breast feeds or oral feeds, 1-10 completed days of postnatal age are eligible for the study.

Exclusion criteria: 1. Clinically ill new-borns (including infections) 2. Major congenital malformations 3. Babies admitted in neonatal intensive care. 4. Preterm neonates. 5. Extremely low birth weight neonates 6 . Babies born with sequele or evidence of congenital infection 7 . Neonates born to mothers with anyone of the following: fever, foul smelling liquor, prolonged rupture of membranes $>12 \mathrm{hrs}$, Lecucocytosis or evidence of documented urinary tract infection within 2 weeks before delivery. 8. Neonates with murmur or suspected to have congenital heart disease.

Participants: Term neonates born at Government Rajaji hospital, Madurai less than 11 days old meeting the inclusion criteria are enrolled.

Outcome Variables: 1. The mean, median, percentile values of PI and PVI in the first 10 days of life. 2. Comparison of mode of delivery with PI and PVI. 3. Comparison of PI and PVI values between $<3$, 3-7 and $>7$ days of life. 4. Correlation of PI, PVI with birth weight and pulse rate.

Study size: Convenient sample of 300 was taken.

Procedure: All the eligible neonates are enrolled after informed consent. A focused history using a structured proforma and clinical examination was done. The peripheral and central body temperature is assessed by touching the back of the hand. The Pulse oximeter used was masimo radical 7 monitor with a reusable neonatal probe. The Pulseoximeter probe is placed randomly on the right or left foot of the neonate using a Velcro type wrap. Once the Pulseoximeter started showing adequate signal and readings, the start time as displayed in the Pulseoximeter is recorded manually in the proforma. The date of Last menstrual period in a mother with regular periods is used for calculating gestational age. The Saturation, Heart rate, PI and PVI values are continuously recorded for next 15 minutes and the end time was also noted in the proforma. The Pulseoximeter recordings are stored in the memory of the Pulseoximeter. Most of the recordings were done after a post fed state and if a baby cries or shows ques for feeding, during recording, the mother was allowed to feed.

Exit criteria: When there is loosening of probe or technical fault during recording and the mother is more concerned about repeat recording, that baby is excluded. When there is a disparity in gestational age assessment found later after recording, those babies data are excluded from analysis. 
Analysis: The data is transferred to a computer using a data transfer software (Masimo Trend).The data transfers itself as a excel sheet. Using the time period of recording, the individual patient's data is retrieved and used for analysis. The pulseoximeter used has an averaging time of 8 seconds. When the Pulseoximeter alarm messages displayed in the excel sheet show low perfusion, low IQ signal, sensor off, ambient light, then the corresponding saturation, Pulse rate, PI, PVI values are excluded. Those readings which does not have all the four values sao 2, heart rate, PI, PVI are excluded.
Statistical methods: Analysis was performed using the statistical software SPSS 16.0. Categorical variables were analyzed with Chi-square or Fisher's exact test.

Continuous variables were analyzed with independent samples test or Mann-Whitney U test.

Results were defined as statistically significant when the $\mathrm{P}$ value (2-sided) was less than 0.05. Pearson's Correlation co-efficient (r) was used to study correlation.

\section{Results}

300 neonates were enrolled in the study after informed consent. Totally Fifteen neonates excluded from the study, six due to probe loosening and malfunction. Another 9 babies were withdrawn since their mothers were more concerned about the 15 minutes recording time, which was not a routine practice prior to the study in the hospital. The data of eight neonates were excluded because of frequent alarm messages displayed as mentioned in the protocol. Totally the data of 277 neonates were included for analysis

Table-1: Baseline Characteristics.

\begin{tabular}{|c|c|c|}
\hline Study Population characteristics & Mean (SD) & N (\%) \\
\hline Maternal Age (in yrs.) & $24.0 \pm 3.9$ & \\
\hline Parity & & $151(54.5)$ \\
\hline Multi & & $126(45.5)$ \\
\hline Primi & & $86(31.0)$ \\
\hline Mode of Delivery & & $183(66.1)$ \\
\hline LN & & $2(0.7)$ \\
\hline LSCS & & $4(1.4)$ \\
\hline Breech & & $2(0.7)$ \\
\hline Forceps & & $277(100.0)$ \\
\hline Vacuum & & \\
\hline Gestational Period & & $156(56.3)$ \\
\hline Term & & $121(43.7)$ \\
\hline Gender & & \\
\hline Male & $3.5 \pm 2.3$ & \\
\hline Female & $2.7 \pm 0.4$ & $6(2.2)$ \\
\hline Age (in days) & & \\
\hline Birth Weight (in kg) & & \\
\hline Site & & $271(97.8)$ \\
\hline Right Foot & & \\
\hline Left Foot & & \\
\hline
\end{tabular}

The mean maternal age was 24.0, SD (3.9).About 54.5\% of the study population were born to multi parous women and $45.5 \%$ were primi mothers. $66.1 \%$ of the study population were Lscs born and $31.0 \%$ were born labour naturale.Other mode of delivery constitutes $2.8 \%$. The mean age of neonates studied was 3.5 days (SD 2.3) and $56.3 \%$ were male neonates and $43.7 \%$ females. (Table 1).

The Primary outcome, median value of perfusion index is $1.4 \mathrm{IQR}(1.0,2.0)$ and pleth variability index is 19.0 , IQR $(15.0,25.0)$. (Table 2) The 5th and 95th percentile values for perfusion index is 0.6 and 3.9 and for PVI it is $10.4 \%$ and $95 \%$ respectively. (Table 3) 
Table-2: Median IQR values of PI and PVI in healthy term neonates in first 10 days of life.

\begin{tabular}{|c|c|c|c|}
\hline & N & Mean \pm SD & Median (IQR) \\
\hline PI & 277 & $1.7 \pm 1.1$ & $1.4(1.0,2.0)$ \\
\hline PVI & 277 & $20.3 \pm 6.6$ & $19.0(15.0,25.0)$ \\
\hline
\end{tabular}

*PI and PVI is non normal distribution. Kolmogorov-Smirnov test was used to test the normality.

Table-3: Percentile values for PI and Pvi in healthy term neonates in the first 10 days of life.

\begin{tabular}{|c|c|c|c|c|c|c|c|}
\hline Percentile & 5 & 10 & 25 & 50 & 75 & 90 & 95 \\
\hline PI & 0.6 & 0.7 & 1 & 1.4 & 2 & 3.1 & 3.9 \\
\hline PVI & 10.4 & 12 & 15 & 19 & 25 & 30 & 33 \\
\hline
\end{tabular}

Table-4: Comparison of Mode of Delivery with Median values (inter-quartile range) of PI, PVI.

\begin{tabular}{|c|c|c|c|}
\hline \multirow{2}{*}{ Variables } & \multicolumn{2}{|c|}{ Mode of Delivery } & \multirow{2}{*}{ P value* $^{*}$} \\
\cline { 2 - 4 } & $\mathbf{L N}$ & $\mathbf{( n = 1 8 3 )}$ & \multirow{2}{*}{0.026} \\
\cline { 2 - 4 } & $1.3(0.8,2.0)$ & $1.5(1.1,2.1)$ & 0.07 \\
\hline PI & $19.0(15.0,23.0)$ & $21.0(16.0,25.0)$ & $0.076)$ \\
\hline
\end{tabular}

*Mann-Whitney U Test. There is a significant difference in PI between labour natural and caesarean deliveries.

Table-5: Comparison of different age group with Median values (inter-quartile range) of PI, PVI

\begin{tabular}{|c|c|c|c|c|}
\hline \multirow{2}{*}{ Variables } & \multicolumn{3}{|c|}{ Age (in days) } & \multirow{2}{*}{ P value* } \\
\cline { 2 - 5 } & $<\mathbf{3}$ & $\mathbf{3}-\mathbf{7}$ & $\mathbf{7}$ & \\
\cline { 2 - 5 } & $(\mathbf{n = 1 2 5})$ & $\mathbf{( n = 1 3 0 )}$ & $1.3(0.9,2.1)$ & 0.693 \\
\hline PI & $1.4(0.9,1.9)$ & $1.5(1.0,2.1)$ & $17.5(12.0,19.2)$ & 0.051 \\
\hline PVI & $20.0(16.0,26.0)$ & $20(15.0,24.0)$ & $\mathbf{( 2 2 )}$ & \\
\hline
\end{tabular}

*Kruskal-Wallis Test

Table-6: Correlation of PI and PVI with birth weight and pulse rate in healthy term neonates in first 10 days of life.

\begin{tabular}{|c|c|c|c|c|c|}
\hline & PI vs. PVI & PR vs. PI & PR vs. PVI & $\begin{array}{c}\text { PVI vs. Birth } \\
\text { Weight ( kg) }\end{array}$ & $\begin{array}{c}\text { PI vs. Birth } \\
\text { Weight( kg) }\end{array}$ \\
\hline $\begin{array}{c}\text { Correlation co- } \\
\text { efficient r (*) }\end{array}$ & 0.196 & 0.023 & 0.034 & 0.088 & 0.086 \\
\hline P-value & $0.001^{* *}$ & 0.699 & 0.569 & 0.142 & 0.151 \\
\hline
\end{tabular}

PR - Pulse rate/minute.

*Karl Pearson's correlation co-efficient

**Significant Weak positive correlation between perfusion index and pleth variability index.

The Perfusion index is significantly lower in neonates delivered labour natural (median 1.3) compared to caesarean deliveries (median 1.5). but the pleth variability index didnot show significant difference between the two modes of delivery. we could not find any significant difference in the above indexes among babies less than 3 days, 3-7 days and 7-10 days of life. (Table 4, 5). The perfusion index and PVI showed a significant weak positive correlation in the present study whereas there is no significant correlation of PI and PVI with pulserate and birth weight. (Table 6) 


\section{Discussion}

Our study reports the reference ranges for perfusion index and pleth variability index in healthy term Indian neonates in the first 10 days of life. We measured the post ductal PI, PVI values, like other studies $[4,5]$. We measured the indices starting from $24 \mathrm{hrs}$ of life to avoid immediate transitional changes at birth and the need to observe the baby in the initial hours for respiratory distress and to establish feeds.

The median value of Perfusion index reported in the present study is $1.4 \mathrm{IQR}(1.0,2.0)$ is similar to another study in 2,032 newborns reporting a preductal median of 1.6 IQR (1.2-2.3), and post ductal median of 1.4 with IQR( $1-2)(p<0.001)$ [8]. But our values are lesser when compared to a large study which reported a post ductal median equal to 1.71 with interquartile range of $1.20-2.5$ in 10,000 healthy newborns between 1 and 120 hours of age [12]. Further compared to our values, the median PI reported by a study in the first 10 minutes of life was much higher 2.1, (IQR 1.4-3.5) [10] whereas the median (IQR) PI for very preterm neonate reported by another study was low $0.70(0.29-1.35)$. [11]

The median value of PVI reported in the present study 19.0, [IQR $(15.0,25.0)]$ is lower than the first day median PVI values 20\% [IQR 15 - 24\%] reported [5]. But in preterm neonates in the first postnatal week, a higher value of median PVI 22, (IQR 18-27) was reported by another study [13].

In our study 16 neonates had an average Perfusion Index of 0.6 (less than 0.7 ). This comprises $5.77 \%$ of the study population. Another study reported $8 \%$ of the healthy term newborns in their study had a perfusion index less than 0.7 [8]. Some studies report a higher preductal PI than post ductal PI (mean difference $0.25 \pm 1$ ); others report a higher post ductal PI. We did not measure the preductal values. We find significantly low Perfusion index in neonates born natural labour compared to caesarean deliveries but other studies found no difference[5]. A pilot study in newborn infants during surgery [14] and a large metanalysis in all ages found PVI has a reasonable ability to predict fluid responsiveness [7], whereas another recent study comparing PVI and Doppler-based stroke volume for detecting fluid responsiveness found none of the algorithms showed a good ability to predict fluid responsiveness [15]. Our study will provide a baseline normative reference data for future studies in this population

\section{Conclusion}

Perfusion index (PI) and PVI are dynamic variables that changes during recording, due to change in local microvascular blood flow. Recording over a period of time (15 minutes) and averaging them in the present study overcome the bias in recording. Also direct data transfer to computer, avoiding observer bias and recording errors are the strengths of our study.

Limitations are, the trend over changes in PI and PVI in the same neonate are not determined, since this is not a longitudinal followup study. We did not record the skin temperature, blood pressure and capillary refill time, at the time of PI measurements. Also variability in the first 24 hours of life was not studied. Our study didnot include Preterm neonates, iugr babies less than $1 \mathrm{~kg}$, well babies with risk factors for sepsis. We recommend further studies in the above risk population and in other centers for validation

Contribution: DR. J.A involved in study design \& data collection, Dr. S.B guided study design and corrected manuscript, Dr.K.M monitored the study and revised manuscript.

\section{What this study adds?}

Perfusion index and plethvariabity index are noninvasive measures of perfusion.

First time we report reference ranges in Indian term neonates less than 10 days of life.

The median value of Perfusion index is $1.4 \mathrm{IQR}$ $(1.0,2.0)$ and the median value of PVI reported in the present study is 19.0 , [IQR $(15.0,25.0)$.

Acknowledgement: We sincerely thank NICU Doctors and Postgraduates, Parents of neonates and Obstetric Department, Govt Rajaji Hospital, Madurai.

\section{Declarations}

Funding: Nil

Conflict of interest: Nil

Institutional Review Board approval: Yes 


\section{References}

1. Goldman JM, Petterson MT, Kopotic RJ, Barker SJ. Masimo signal extraction pulse oximetry. J Clin Monit Comput. 2000;16(7):475-83.

2. Corp M. Technical Bulletin Pleth Varibility Index [Internet]. White Papers. [cited 2017July21]. Available from: http://www.masimo.com/de/ Home/ clinical-evidence/white-papers/

3. Lima AP, Beelen P, Bakker J. Use of a peripheral perfusion index derived from the pulse oximetry signal as a noninvasive indicator of perfusion. Crit Care Med. 2002 Jun;30(6):1210-3.

4. Zaramella P, Freato F, Quaresima V, Ferrari M, Vianello A, Giongo D, Conte L, Chiandetti L. Foot pulse oximeter perfusion index correlates with calf muscle perfusion measured by near-infrared spectroscopy in healthy neonates. J Perinatol. 2005 Jun;25(6):417-22.

5. Latini G, Dipaola L, De Felice C. First day of life reference values for pleth variability index in spontaneously breathing term newborns. Neonatology. 2012;101(3):179-82. doi: 10.1159/000 331774. Epub 2011 Oct 22.

6. De Felice C, Latini G, Vacca P, Kopotic RJ. The pulse oximeter perfusion index as a predictor for high illness severity in neonates. Eur J Pediatr. 2002 Oct;161(10):561-2. Epub 2002 Sep 3.

7. Chu H, Wang Y, Sun Y, Wang G. Accuracy of pleth variability index to predict fluid responsiveness in mechanically ventilated patients: a systematic review and meta-analysis. J Clin Monit Comput. 2016 Jun; 30 (3):265-74. doi: 10.1007/ s10877- 015 -9742-3. Epub 2015 Aug 5.

8. Jardim J, Rocha R, Silva G, Guimarães H. Peripheral perfusion index-reference range in healthy Portuguese term newborns. J Pediatr Neonat Individual Med. 2014;3(1):e030109. doi: 10.7363/ 030109
9. Hakan N, Dilli D, Zenciroglu A, Aydin M, Okumus N. Reference values of perfusion indices in hemodynamically stable newborns during the early neonatal period. Eur J Pediatr. 2014 May;173(5): 597-602. doi: 10.1007/s00431-013-2224-z. Epub 2013 Dec 3.

10. Kroese JK, van Vonderen JJ, Narayen IC, Walther FJ, Hooper S, te Pas AB. The perfusion index of healthy term infants during transition at birth. Eur J Pediatr. 2016 Apr;175(4):475-9. doi: 10.1007/s00431-015-2650-1. Epub 2015 Oct 24.

11. Kinoshita M, Hawkes CP, Ryan CA, Dempsey EM. Perfusion index in the very preterm infant. Acta Paediatr. 2013 Sep;102(9):e398-401. doi: 10.1111/ apa. 12322 .

12. Granelli Ad, Ostman-Smith I. Noninvasive peripheral perfusion index as a possible tool for screening for critical left heart obstruction. Acta Paediatr. 2007 Oct;96(10):1455-9. Epub 2007 Aug 28. PubMed PMID: 17727691.

13. Vidal M, Ferragu F, Durand S, Baleine J, Batista-Novais AR, Cambonie G. Perfusion index and its dynamic changes in preterm neonates with patent ductus arteriosus. Acta Paediatr. 2013 Apr;102(4):373-8. doi: 10.1111/apa.12130. Epub 2013 Jan 19.

14. Bagci S, Müller N, Müller A, Heydweiller A, Bartmann P, Franz AR. A pilot study of the pleth variability index as an indicator of volumeresponsive hypotension in newborn infants during surgery. J Anesth. 2013 Apr;27(2):192-8. doi: 10. 1007/s00540-012-1511-6. Epub 2012 Nov 7. Pub Med PMID: 23132180.

15. Bahlmann H, Hahn RG, Nilsson L. Agreement between Pleth Variability Index and oesophageal Doppler to predict fluid responsiveness. Acta Anaesthesiol Scand. 2016 Feb;60(2):183-92. doi: 10. 1111/aas.12632. Epub 2015 Sep 16.

\section{How to cite this article?}

Ashok Raja J, Balasankar S, Mathiarasan K. Perfusion index and Plethysmographic variability index values in spontaneously breathing clinically stable term neonates in room air in the first 10 days of life. J PediatrRes. 2017;4(08):531-536.doi:10. 17511/ijpr.2017.i08.06. 